\section{Christoph Bachmann}

\section{Einleitung}

Stress kann bei den Betroffenen eine Kaskade von psychischen Symptomen wie Reizbarkeit, Angst und Konzentrationsmangel auslösen. Ebenso können physische Symptome wie Müdigkeit oder Erschöpfung beobachtet werden. Untersuchungen zeigen, dass $50-60 \%$ der krankheitsbedingten Arbeitsausfälle auf Stress zurückzuführen sind, wobei Frauen hiervon mehr betroffen sind als Männer [1]. Stress kann aber in Betrieben und Organisationen in allen Bereichen und auf allen Hierarchiestufen für beide Geschlechter ein Problem darstellen.

\section{Rhodiola rosea (Rosenwurz)}

Der Rosenwurz ist eine adaptogene Arzneipflanze. Studien deuten darauf hin, dass er die Resistenz gegen Stress, Traumen, Ängstlichkeit, Erschöpfung und Müdigkeit erhöht. Der Wirkungsmechanismus scheint aus einem zentralen und peripheren Eingriff in die Monoamin- und Opoidsynthese $\mathrm{zu}$ bestehen [2].

\section{Anwendungsbeobachtung}

Verschiedene klinische Studien zeigten eine Verbesserung der körperlichen und mentalen Arbeitsleistung, der mentalen Leistung unter Stress [35] sowie unter anderen extremen Bedingungen.

\section{Aus der Forschung}

Schweiz Z Ganzheitsmed 2016;28:197-198

Published online: June 28, 2016

\title{
Therapeutische Wirksamkeit und Sicherheit des Rosenwurz- Extraktes WS 1375: Resultate einer Anwendungsbeobachtung
}

Stress ist eines der ganz grossen Probleme der westlichen Welt. Dabei handelt es sich sehr oft um Folgen einer ungesunden Lebensweise, die durch eine übervolle Agenda sowie durch einen Mangel an sinnvoll gestalteter, erholsamer Freizeit entsteht. Daneben kommt Stress aber auch bei Menschen vor, die unfreiwillig unter Druck stehen, wofür viele verschiedene Ursachen infrage kommen.

Eine Anwendungsbeobachtung wurde angelegt, um die Wirksamkeit des Rosenwurz-Extraktes WS ${ }^{\circledR} 1375$ (Wirkstoff von Vitango ${ }^{\circledR}$, Schwabe GmbH in der Schweiz: Vitango ${ }^{\circledR}$, Schwabe Pharma AG, Küssnacht a.R.) in einer vierwöchigen Anwendung bei Personen mit Stresssymptomen zu untersuchen.

\section{Studiendesign}

Die Studie wurde als nichtrandomisierte, offene, einarmige Multizenterstudie angelegt und schloss 30 - bis 60-jährige Probanden ein, die auf einer Visuellen Analogskala (VAS) bei mindestens 3 von 7 möglichen Stresssymptomen die Intensität der Symptome mit mindestens 5 angegeben hatten. Bei Baseline unterschieden sich die Werte der Probanden nicht wesentlich.

Diese Probanden erhielten während 4 Wochen zweimal täglich 1 Tablette WS 1375 à 200 mg Extrakt. Von den ursprünglich 109 rekrutierten Probanden konnten 82 in die Per-Protocol-Auswertung aufgenommen werden. Alle Dropouts wurden dokumentiert.

\section{Zielvariablen}

Als Zielvariablen wurden die auf einer VAS bestimmten Werte von 7 verschiedenen Stresssymptomen verwendet. Diese waren: körperliche Symptome, Verlust der Lebensfreude, Erschöpfung, Reizbarkeit, Konzentrationsmangel, Gefühl der Fremdbestimmtheit und Ängstlichkeit.

Als weitere Stressparameter wurden der Perceived Stress Questionnaire (PSQ), das Multidimensional Fatigue Inventory (MFI-20), der Numbers Connecting Test (NCT), der Sheehan Disability Scale, die englische Version des Multidimensional Mood State Questionnaire (MDMQ) sowie die Clinical Global Impression (CGI) zugezogen.

Die Sicherheit des Studienpräparates wurde anhand verschiedener Tests überprüft.

\section{Resultate}

\section{VAS}

Bei den festgehaltenen Werten bezüglich der Stresssymptome wurde schon nach 3 Tagen eine Verbesserung von $1,5 \pm 2,5$ auf $2,2 \pm 2,5$ Skalenpunkte ermittelt.

\section{KARGER}

๑) 2016 S. Karger GmbH, Freiburg 
Nach 1 Woche verbesserten sich diese Werte auf 2,1 $\pm 2,8$ bis $3,2 \pm 2,7$. Am Ende der Studie hatten diese Werte $2,8 \pm 3,0$ bis $3,7 \pm 4,3$ Punkte erreicht. Bei jeder dieser 3 Auswertungen bedeutete dies eine verglichen mit der Baseline signifikante Verbesserung $(\mathrm{p}<0,0001)$.

$$
\text { PSQ }
$$

Auch bei diesem Parameter wurde zwischen Baseline und dem Studienende eine signifikante Verbesserung gemessen, d.h. Baseline 1,5 $\pm 1,4$ versus Studienende 3,8 $\pm 4,3(\mathrm{p}<0,0001)$.

\section{Sheehan Disability Scale}

Bei den 3 Bereichen Arbeit/Schule, soziale Aspekte sowie Familie/private Verantwortungen wurde im Verlauf der Studie eine signifikante Verbesserung festgestellt $(\mathrm{p}<0,0177 \mathrm{bis}<0,0001)$.

\section{CGI}

Bereits 3 Tage nach Studienbeginn konnte beim Gesamtzustand der Probanden eine signifikante Verbesserung festgestellt werden. Dieser verbesserte sich im Verlauf der Studie weiter und blieb auch nach 1 sowie nach 4 Wochen signifikant.

\section{Weitere Tests}

Auch die ermittelten Verbesserungen beim MFI-20, NCT und MDMQ zeigten zu jedem der ermittelten Zeitpunkte in der Studie jeweils Signifikanz, mit Ausnahme des Items «Reduzierte Aktivität» im MFI-20.

\section{Sicherheit}

Im Rahmen der Studie wurden verschiedene milde bis moderate, jedoch keine schwerwiegenden unerwünsch- ten Wirkungen dokumentiert. Diese betrafen vor allem Nervosität und gastrointestinale Beschwerden.

\section{Fazit und Schlussfolgerung}

Die vierwöchige Anwendungsbeobachtung dokumentiert die Wirksamkeit und Sicherheit des Extraktes WS 1375 aus Rhodiola rosea bei Erwachsenen mit Stresssymptomen, die auf den Lebensstil zurückzuführen sind. Alle ermittelten Resultate zeigten eine verschiedene Symptome betreffende körperliche und mentale Wirksamkeit, die - mit einer Ausnahme - schon 3 Tage nach Studienbeginn signifikant war.

Damit erweist sich WS 1375 in einer Dosierung von zweimal täglich $200 \mathrm{mg}$ als wirksames und sicheres adaptogenes Therapeutikum zur Behandlung von lebensstilbedingten Stresssymptomen.

\section{Literatur}

1 Milczarek M, Schneider E, Gonzales ER: OSH in Figures: Stress at Work - Facts and Figures. Luxembourg, European Agency for Safety and Health at Work, Office for Official Publications of the European Communities, 2009, pp 20-28.

$\checkmark 2$ Kelly GS: Rhodiola rosea: a possible plant adaptogen. Altern Med Rev 2001;6:293-302.

3 Shevtsov VA, Zholus BI, Shervarly VI, et al: A randomized trial of two different doses of a SHR-5 Rhodiola rosea extract versus placebo and control of capacity for mental work. Phytomedicine 2003;10:95-105.

4 De Bock K, Eijnde BO, Ramaekers M, Hespel P: Acute Rhodiola rosea intake can improve endurance exercise performance. Int J Sport Nutr Exerc Metab 2004;14:298-307.
Spasov AA, Wikman GK, Mandrikov VB, Mironova IA, Neumoin VV: A double-blind, placebo-controlled pilot study of the stimulating and adaptogenic effect of Rhodiola rosea SHR-5 extract on the fatigue of students caused by stress during an examination period with a repeated low-dose regimen. Phytomedicine 2000;7:85-89. 